12,01

\title{
Атомная подвижность в тройном жидком сплаве Ga-In-Sn эвтектического состава
}

\author{
() Д.Ю. Нефедов ${ }^{1}$, А.О. Антоненко ${ }^{1}$, Д.Ю. Подорожкин ${ }^{1}$, A.В. Усков ${ }^{1}$, Е.В. Чарная ${ }^{1, \uparrow}$, M.K. Lee ${ }^{2}$, \\ J.L. Chang ${ }^{3}$, J. Haase ${ }^{4}$, D. Michel ${ }^{4}$, Ю.A. Кумзеров ${ }^{5}$, A.B. Фокин ${ }^{5}$, М.И. Самойлович ${ }^{6}$, А.C. Бугаев $^{7}$
}

${ }^{1}$ Санкт-Петербургский государственный университет,

Санкт-Петербург, Россия

${ }^{2}$ NSC Instrument Center at NCKU,

Tainan, Taiwan

${ }^{3}$ Department of Physics, National Cheng Kung University,

Tainan, Taiwan

${ }^{4}$ Faculty of Physics and Geosciences, Leipzig University,

Leipzig, Germany

${ }^{5}$ Физико-технический институт им. А.Ф. Иофффе РАН,

Санкт-Петербург, Россия

${ }^{6}$ ОАО ЦНИТИ „Техномаш“,

Москва, Россия

${ }^{7}$ Московский фризико-технический институт (Государственный университет),

Долгопрудный, Россия

ฯ E-mail: charnaya@mail.ru

(Поступила в Редакцию 22 июня 2016 г.)

Проведены исследования ядерной спин-решеточной релаксации и сдвига Найта ядер ${ }^{71} \mathrm{Ga},{ }^{69} \mathrm{Ga}$ и ${ }^{115} \mathrm{In}$ в жидком тройном сплаве галлия, индия и олова эвтектического состава, введенном в поры опаловой матрицы и пористых стекол с размером пор 18 и $7 \mathrm{~nm}$, в сравнении с объемным расплавом. Обнаружено ускорение продольной релаксации и уменьшение сдвига Найта, зависящие от размера пор. Рассчитано время корреляции атомного движения для наноструктурированного расплава в пористых матрицах. Показано, что атомная подвижность в расплаве понижается с уменьшением размера пор.

DOI: $10.21883 /$ FTT.2017.02.44062.254

\section{1. Введение}

В последнее время значительно вырос интерес к исследованию тройного сплава галлия, индия и олова (GaInSn) в связи с расширяющейся областью его практических применений. Как и другие тройные сплавы, сплав GaInSn изучен значительно меньше, чем бинарные сплавы металлов. Сплав GaInSn имеет фазовую диаграмму эвтектического типа [1]. Эвтектическая точка соответствует составу 75, 17 и 8 at.\%. На практике используется сплав под названием „галинстан“, в котором концентрация компонент несколько отличается от приведенной выше за счет присутствия небольшого количества других элементов. Галинстан широко применяется в производстве безртутных термометров, в качестве рабочих электродов в вольтамперметрии, при производстве жидких металлических теплоносителей, а также в биотехнологиях [2,3]. Кроме того, сплав GaInSn перспективен для использования в нанокомпозитных системах, в частности в ферроколлоидных суспензиях. В связи с этим особенно актуальным становится изучение влияния наноструктурирования на вязкость и атомную диффузию в жидком сплаве GaInSn. Для наноструктурированных бинарных расплавов $\mathrm{Ga}-\mathrm{In}$ и $\mathrm{Ga}-\mathrm{Sn}$ и ряда жидких металлов методами ЯМР было выявлено значительное замедление атомного движения [4-6]. Для тройных сплавов этот вопрос ранее не исследовался.

Целью настоящей работы является исследование методом ЯМР атомной подвижности в жидком сплаве GaInSn эвтектического состава, наноструктурированном за счет введения в три различные нанопористые силикатные матрицы.

\section{2. Образцы и эксперимент}

В качестве нанопористых матриц использовались пористые стекла со средним размером пор $d=7$ и $18 \mathrm{~nm}$, а также опаловая матрица, представляющая собой плотную упаковку шаров из аморфного кремнезема диаметром $210 \mathrm{~nm}$. Пористые стекла изготавливались в лабораторных условиях путем выщелачивания прошедших термообрабоку силикатных натриево-боратных стекол, в результате которого формировался каркас со связанной сеткой пор. Размер пор определялся методами азотной и ртутной порометрии. Диаметр шаров в опаловой матрице находился методом атомно-силовой микроскопии. Между шарами в опаловой матрице имеется регулярная сетка связанных пор двух типов, октаэдрических и тетраэдрических. Размер пор задается геометрией плотной упаковки шаров. Для диаметра шаров $210 \mathrm{~nm}$ окта- 
эдрические и тетраэдрические полости должны иметь размеры около 87 и $47 \mathrm{~nm}$ соответственно. При спекании опаловых матриц, однако, происходит уменьшение размеров пор обоих типов.

Сплав GaInSn эвтектического состава вводился в поры под давлением при комнатной температуре в жидком состоянии. В эвтектической точке сплав плавится при температуре около $285 \mathrm{~K}$ [1]. После заполнения пор поверхность матриц очищалась, и вырезались образцы для исследований объемом около $0.2 \mathrm{~cm}^{3}$. Затем поверхность образцов снова очищалась от остатков объемного сплава. Фактор заполнения пор определялся по взвешиванию до и после заполнения и составлял около $80 \%$ для всех трех образцов. Для сравнения был также приготовлен объемный сплав GaInSn соответствующего состава.

ЯМР измерения проводились при комнатной температуре на трех импульсных спектрометрах фирмы Bruker, Avance 400, MSL 500 и Avance 750, в магнитных полях 9.4, 11.75 и 17.6 Т соответственно. Использование различных спектрометров позволяло выявить зависимости измеряемых характеристик от величины квантующего магнитного поля. Исследовалась скорость продольной спиновой релаксации и сдвиг резонансной линии для двух изотопов галлия, ${ }^{71} \mathrm{Ga}$ и ${ }^{69} \mathrm{Ga}$, и изотопа индия ${ }^{115} \mathrm{In}$. Релаксация измерялась по восстановлению сигнала ядерной намагниченности после инвертирующего $180^{\circ}$-импульса. Положение линии ЯМР определялось по положению максимума сигнала, полученного Фурьепреобразованием сигнала свободной прецессии после $90^{\circ}$-импульса. Из-за слабости сигналов ЯМР для расплава в нанопорах количество накоплений варьировалось от 2 до 16 тысяч. Сдвиг линии ЯМР (сдвиг Найта) для изотопов галлия вычислялся относительно положения резонансной линии в кубическом монокристалле $\mathrm{GaAs}$ [4], а для индия - относительно положения линии в слабом растворе $\mathrm{InNO}_{3}$.

Полученные экспериментальные результаты для спиновой релаксации в совокупности с данными о сдвиге Найта использовались для расчета времени корреляции атомного движения в наноструктурированном и объемном расплаве, что позволило получить информацию о замедлении атомной диффузии в расплаве как следствие наноконфайнмента. Следует подчеркнуть, что широко распространенная для анализа диффузионных процессов в неметаллических жидкостях градиентная техника ЯМР [7] не применима к расплавам металлов из-за коротких времен ядерной спиновой релаксации. Из-за понижения скорости диффузии в результате наноструктурирования становится также неинформативным метод рассеяния нейтронов [8].

\section{3. Экспериментальные результаты}

Линии ЯМР всех исследованных изотопов для сплава, введенного в нанопористые матрицы, значительно уширялись по сравнению с линиями для объемного

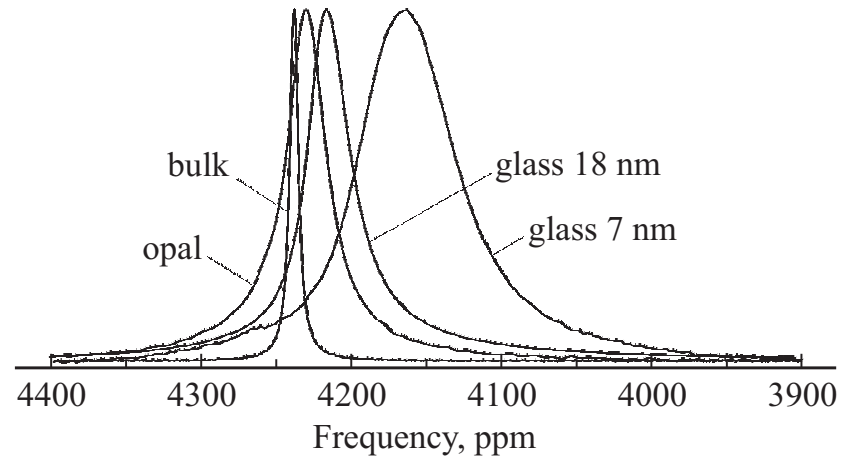

Рис. 1. Линии ЯМР изотопа ${ }^{71} \mathrm{Ga}$, полученные в поле $9.4 \mathrm{~T}$, для исследованных образцов.

сплава. В частности, ширина резонансной линии индия на половине высоты увеличивалась от $23 \mathrm{ppm}$ для объемного сплава до $280 \mathrm{ppm}$ в поле $9.4 \mathrm{~T}$ для сплава, введенного в пористое стекло с размером пор $18 \mathrm{~nm}$. Для обоих изотопов галлия и изотопа индия также происходило смещение резонансных линий в сторону низких частот. Пример линий ЯМР ${ }^{71} \mathrm{Ga}$, полученных в поле 9.4 Т для всех четырех образцов, показан на рис. 1. Как видно, наибольший сдвиг линии ЯМР наблюдается для сплава в стекле с размером пор $7 \mathrm{~nm}$, где сдвиг Найта уменьшается примерно на 80 ppm. Сдвиги Найта для всех исследованных случаев сведены в табл. 1-3, в которых представлены результаты измерений с наименьшей погрешностью. Для индия из-за сильного уширения сигналы были получены только для сплава в пористом стекле с порами $18 \mathrm{~nm}$ и опаловой матрице. Измерения проводились только в полях 11.75 и $9.4 \mathrm{~T}$.

Восстановление продольной ядерной намагниченности для изотопов галлия и индия в объемном расплаве было одноэкспоненциальным также, как и для других объемных жидких металлов и сплавов, и, следовательно, описывалось временем релаксации $T_{1}$ [9]. Скорость релаксации была одинаковой в разных полях в пределах экспериментальной погрешности. Одноэкспоненциальная и независящая от поля релаксация соответствует приближению быстрого движения [10], что также согласуется с узкими резонансными линиями. В результате замедления атомной подвижности при введении расплава в нанопористые матрицы это приближение может нарушаться, как было найдено при исследовании жидких металлов и бинарных сплавов в условиях наноконфайнмента [4-6]. Однако, как показали оценки времени корреляции атомного движения, проведенные ниже в разделе Обсуждение, приближение быстрого движения еще выполняется для сплава GaInSn в стекле с порами $18 \mathrm{~nm}$ и в опале. Для сплава в этих матрицах, так же как и в объемном сплаве, продольная релаксация может быть описана посредством времени релаксации $T_{1}$. Усредненные по нескольким измерениям времена релаксаций приведены в табл. 1-3. Как видно из таблицы, времена релаксации для сплава в стекле с порами $18 \mathrm{~nm}$ 
Таблица 1. Сдвиг Найта $K$, время спин-решеточной релаксации $T_{1}$ и время квадрупольной релаксации $T_{1 Q}$ для ядер ${ }^{71} \mathrm{Ga}$ в исследованных образцах

\begin{tabular}{l|c|c|c|c|c|c|c|c|c|c}
\hline \multicolumn{1}{c|}{ Образец } & Объемный & \multicolumn{3}{|c|}{ Опал } & \multicolumn{3}{c|}{ Стекло $18 \mathrm{~nm}$} & \multicolumn{3}{c}{ Стекло $7 \mathrm{~nm}$} \\
\hline Магнитное & 9.4 & 17.6 & 11.75 & 9.4 & 17.6 & 11.75 & 9.4 & 17.6 & 11.75 & 9.4 \\
поле, T & $4238 \pm 1$ & $4235 \pm 1$ & & & $4231 \pm 3$ & & & $4163 \pm 5$ & \\
$K, \mathrm{ppm}$ & $437 \pm 5$ & $290 \pm 20$ & $286 \pm 10$ & $290 \pm 20$ & $259 \pm 10$ & $265 \pm 10$ & $266 \pm 10$ & $130 \pm 20$ & $120 \pm 20$ & $110 \pm 20$ \\
$T_{1}, \mu \mathrm{s}$ & $2500 \pm 300$ & $570 \pm 80$ & $560 \pm 40$ & $570 \pm 80$ & $470 \pm 30$ & $480 \pm 40$ & $490 \pm 40$ & $170 \pm 30$ & $150 \pm 30$ & $140 \pm 30$
\end{tabular}

Таблица 2. Сдвиг Найта $K$, время спин-решеточной релаксации $T_{1}$ и время квадрупольной релаксации $T_{1 Q}$ для ядер ${ }^{69} \mathrm{Ga}$ в исследованных образцах

\begin{tabular}{|c|c|c|c|c|c|c|c|c|c|c|}
\hline \multirow{2}{*}{\begin{tabular}{l}
\multicolumn{1}{c}{ Образец } \\
$\begin{array}{l}\text { Магнитное } \\
\text { поле, T }\end{array}$
\end{tabular}} & \multirow{2}{*}{$\begin{array}{c}\text { Объемный } \\
9.4\end{array}$} & \multicolumn{3}{|c|}{ Опал } & \multicolumn{3}{|c|}{ Стекло $18 \mathrm{~nm}$} & \multicolumn{3}{|c|}{ Стекло $7 \mathrm{~nm}$} \\
\hline & & 17.6 & 11.75 & 9.4 & 17.6 & 11.75 & 9.4 & 17.6 & 11.75 & 9.4 \\
\hline$K, \mathrm{ppm}$ & $4239 \pm 1$ & $4233 \pm 1$ & & & $4229 \pm 5$ & & & $4159 \pm 10$ & & \\
\hline$T_{1}, \mu \mathrm{s}$ & $483 \pm 5$ & $180 \pm 20$ & $174 \pm 15$ & $164 \pm 15$ & $180 \pm 20$ & $180 \pm 20$ & $170 \pm 20$ & $70 \pm 20$ & $50 \pm 20$ & $50 \pm 20$ \\
\hline$T_{1 Q}, \mu \mathrm{s}$ & $1000 \pm 100$ & $220 \pm 30$ & $210 \pm 20$ & $220 \pm 20$ & $220 \pm 30$ & $220 \pm 20$ & $210 \pm 30$ & $80 \pm 20$ & $60 \pm 20$ & $50 \pm 20$ \\
\hline
\end{tabular}

Таблица 3. Сдвиг Найта $K$, время спин-решеточной релаксации $T_{1}$ и время квадрупольной релаксации $T_{1 Q}$ для ядер ${ }^{115} \mathrm{In}$ в исследованных образцах

\begin{tabular}{|c|c|c|c|c|c|}
\hline Образец & Объемный & \multicolumn{2}{|c|}{ Опал } & \multicolumn{2}{|c|}{ Стекло $18 \mathrm{~nm}$} \\
\hline $\begin{array}{l}\text { Магнитное } \\
\text { поле, T } \\
K, \mathrm{ppm} \\
T_{1}, \mu \mathrm{s}\end{array}$ & $\begin{array}{c}9.4 \\
8431 \pm 1 \\
170 \pm 5\end{array}$ & $\begin{array}{c}11.75 \\
8409 \pm 10 \\
62 \pm 7\end{array}$ & $61 \pm 7$ & $\begin{array}{c}11.75 \\
8401 \pm 10 \\
57 \pm 7\end{array}$ & $\begin{array}{c}9.4 \\
53 \pm 7\end{array}$ \\
\hline$T_{1 Q}, \mu \mathrm{s}$ & 370 & \multicolumn{2}{|c|}{78} & \multicolumn{2}{|c|}{70} \\
\hline
\end{tabular}

и в опале существенно короче, чем в объемном сплаве. Отметим, что, несмотря на увеличение скорости релаксации, времена релаксации изотопов галлия и индия в пределах погрешности не зависят от магнитного поля, что согласуется с приближением быстрого движения. Для иллюстрации уменьшения времени релаксации в условиях наноконфайнмента на рис. 2 и 3 приведены кривые восстановления намагниченности изотопа ${ }^{69} \mathrm{Ga}$ в поле $11.75 \mathrm{~T}$ и намагниченности индия в полях 9.4 и $11.75 \mathrm{~T}$ для объемного и наноструктурированного сплава.

Для жидкого сплава GaInSn в пористом стекле с порами $7 \mathrm{~nm}$ продольная релаксация для обоих изотопов галлия была намного быстрее, чем в объемном сплаве. Кроме того, скорость восстановления намагниченности зависела от магнитного поля, что указывает на невыполнение приближения быстрого движения. Зависимость от поля иллюстрируется на рис. 4 для изотопа ${ }^{71} \mathrm{Ga}$.

Как обсуждается ниже, при нарушении приближения быстрого движения восстановление ядерной намагниченности нельзя описать одной экспонентой и соответственно временем релаксации $T_{1}$ [11]. Для обоих изотопов галлия, имеющих спин, равный $3 / 2$, релаксация описывается суммой двух экспонент. Однако, как было показано в работе [12], кривые восстановления остаются близки к экспоненциальным и могут быть приблизительно охарактеризованы временами релаксации, что облегчает сравнение с релаксацией в объемном сплаве. Времена, сопоставляемые экспериментальным кривым восстановления намагниченности, также приведены в табл. 1 и 2.

\section{4. Обсуждение}

Для интерпретации экспериментальных результатов по влиянию наноструктурирования сплава GaInSn на продольную релаксацию рассмотрим сначала механизмы релаксации в объемных жидких металлах [9]. Релаксация происходит за счет двух основных аддитивных взаимодействий: взаимодействия ядерных магнитных диполей с электронами проводимости и взаимодействия электрических квадрупольных моментов с динамическими градиентами электрических полей, возникающих при 


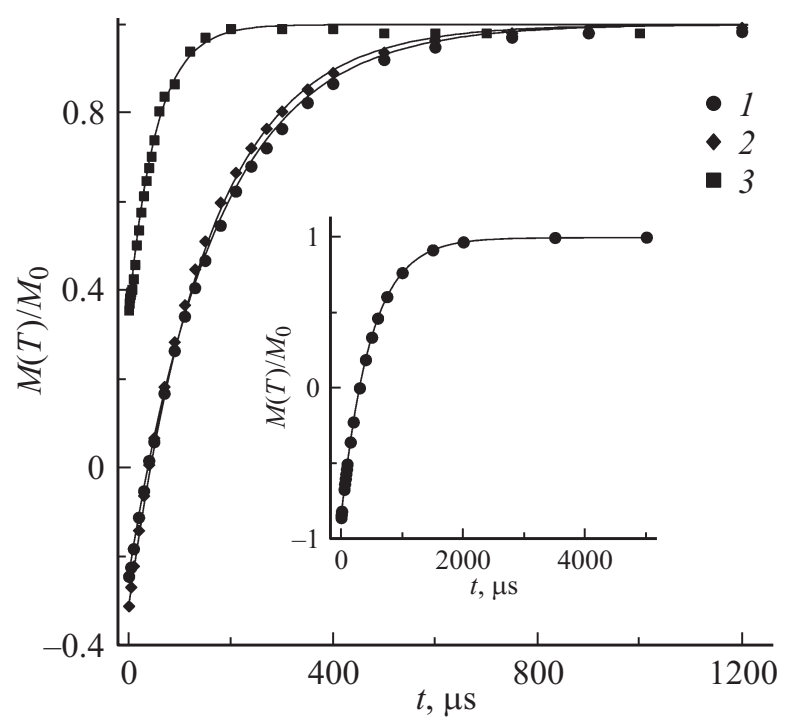

Рис. 2. Кривые восстановления относительной намагниченности $M(t) / M_{0}(M(t)$ - намагниченность как функция времени $t$, $M_{0}$ - равновесная намагниченность) ядер ${ }^{69} \mathrm{Ga}$ после инвертирующего импульса, полученные в поле $11.75 \mathrm{~T}$, для расплава в опаловой матрице (1), в стекле с порами $18 \mathrm{~nm}(2)$ и стекле с порами $7 \mathrm{~nm}$ (3). Сплошные линии - теоретический расчет. На вставке показано восстановление намагниченности ядер ${ }^{69} \mathrm{Ga}$ в объемном расплаве.

движении в жидкости. Магнитная релаксация вносит основной вклад. Приближение быстрого движения справедливо как для магнитной, так и для квадрупольной спиновой релаксации. В итоге восстановление продольной намагниченности описывается одной экспонентой со временем релаксации $T_{1}$, которое удовлетворяет соотношению

$$
\left(T_{1}\right)^{-1}=\left(T_{1 m}\right)^{-1}+\left(T_{1 Q}\right)^{-1},
$$

где $T_{1 m}$ и $T_{1 Q}-$ времена магнитной и квадрупольной релаксации. Для двух изотопов галлия квадрупольный и магнитный вклады связаны между собой следующими соотношениями

$$
\begin{gathered}
T_{1 Q}^{69}=T_{1 Q}^{71} Q_{71}^{2} / Q_{69}^{2}, \\
T_{1 m}^{69}=T_{1 m}^{71} \gamma_{71}^{2} / \gamma_{69}^{2},
\end{gathered}
$$

где $Q$ и $\gamma$ квадрупольный момент и гиромагнитное отношение. Использование соотношений (1)-(3) позволяет рассчитать времена магнитной и квадрупольной релаксации изотопов галлия для объемного сплава GaInSn. Pacчеты дали следующие значения для времени магнитной релаксации: $T_{1 m}^{71}=580 \pm 10 \mu \mathrm{s}$ и $T_{1 m}^{69}=937 \pm 17 \mu \mathrm{s}$. Времена квадрупольной релаксации приведены в соответствующих столбцах табл. 1 и 2. Квадрупольные моменты и гиромагнитные отношения изотопов галлия принимались равными: $\gamma_{71}=8.18 \cdot 10^{7} \mathrm{rad} \cdot \mathrm{T}^{-1} \cdot \mathrm{s}^{-1}, \gamma_{69}=$ $=6.44 \cdot 10^{7} \mathrm{rad} \cdot \mathrm{T}^{-1} \cdot \mathrm{s}^{-1}, Q_{71}=0.107 \mathrm{~b}$ и $Q_{69}=0.171 \mathrm{~b}$.

Проведем теперь анализ релаксации для изотопов галлия в пористом стекле с порами $18 \mathrm{~nm}$ и в опаловой матрице. С хорошей точностью можно полагать, что для жидкого сплава в порах скорость магнитной релаксации не изменилась по сравнению с объемным расплавом. Действительно, время магнитной релаксации и сдвиг Найта $K$ удовлетворяют соотношению Корринги $T_{1 m} T K^{2}=$ const [10], где $T-$ температура, а константа в

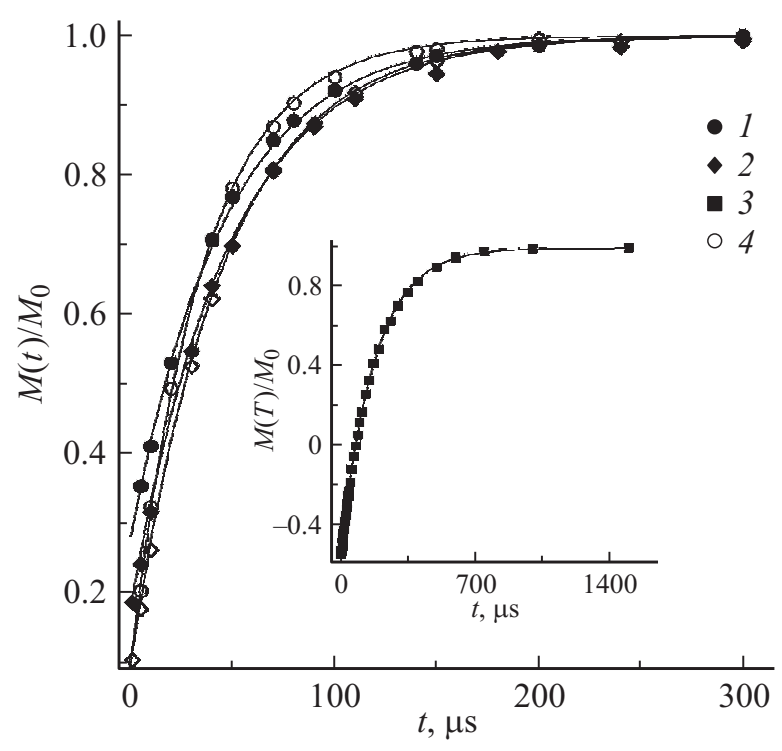

Рис. 3. Кривые восстановления относительной намагниченности $M(t) / M_{0}(M(t)$ - намагниченность как функция времени $t, M_{0}-$ равновесная намагниченность) ядер ${ }^{115}$ In после инвертирующего импульса, полученные в поле $11.75 \mathrm{~T}$ (1) и $9.4 \mathrm{~T}$ (2), для расплава в опаловой матрице (3) и в стекле с порами $18 \mathrm{~nm}$ (4). Сплошные линии - теоретический расчет. На вставке показано восстановление намагниченности ядер ${ }^{115}$ In в поле 9.4 Т для объемного расплава.

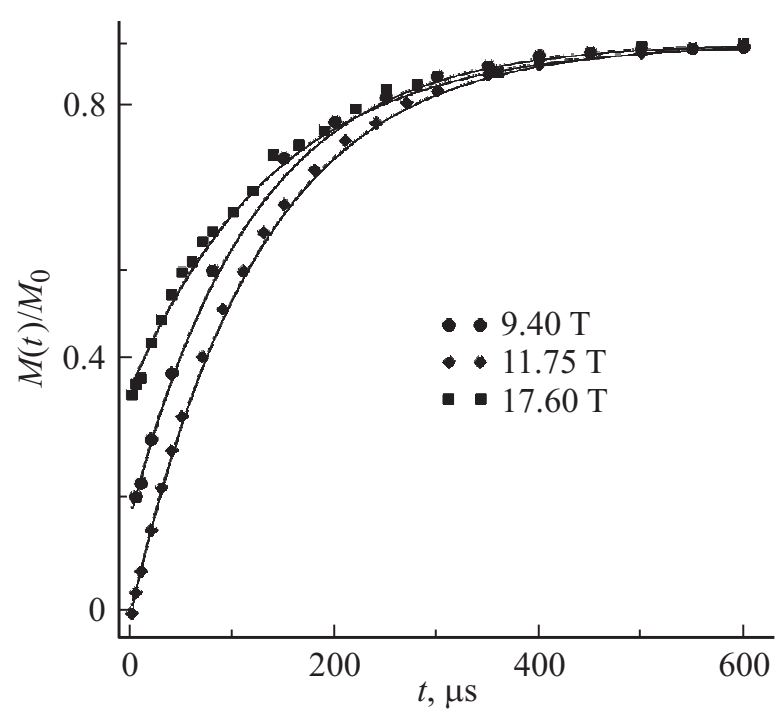

Рис. 4. Кривые восстановления относительной намагниченности $M(t) / M_{0}(M(T)$ - намагниченность как функция времени $t, M_{0}$ - равновесная намагниченность) ядер ${ }^{71} \mathrm{Ga}$ после инвертирующего импульса, полученные в различных полях, для расплава в стекле с порами $7 \mathrm{~nm}$. Сплошные линии теоретический расчет. 
Таблица 4. Квадрупольная константа $C^{71}$, время корреляции атомного движения $\tau_{c}$ и отношение времен корреляции атомного движения в сплаве, введенном в нанопористые матрицы, и объемном сплаве $\tau_{c} / \tau_{c}^{\text {bulk }}$

\begin{tabular}{c|c|c|c|c}
\hline Образец & Объемный & Опал & Стекло $18 \mathrm{~nm}$ & Стекло $7 \mathrm{~nm}$ \\
\hline$C^{71}, \mu \mathrm{s}$ & 28 & 28 & 28 & 31 \\
$\tau_{c}, 10^{-6} \mu \mathrm{s}$ & 14 & 67 & 75 & 230 \\
$\tau_{c} / \tau_{c}^{\text {bulk }}$ & 1 & 4.8 & 5.4 & 16
\end{tabular}

правой части содержит физические постоянные, ядерное гиромагнитное отношение и коэффициент порядка единицы, который учитывает эффекты корреляции электронов. Поскольку сдвиг Найта относительно слабо изменяется при введении сплава GaInSn в пористые матрицы (см. табл. 1 и 2), то можно считать, что наноструктурирование сплава не сказывается существенно на времени магнитной релаксации. Это позволяет рассчитать время квадрупольной релаксации, используя соотношение (1). Результаты расчетов также приведены в табл. 1 и 2 . Как видно из этих таблиц, время квадрупольной релаксации изотопов галлия сокращается в несколько раз по сравнению с временем квадрупольной релаксации в объемном расплаве. Отметим, что слабое уменьшение сдвига Найта в пористых матрицах наблюдалось и для бинарного расплава галлия и индия [13].

Отметим, что разделить вклады магнитной и квадрупольной релаксации для сплава в опале и пористом стекле с размером пор $18 \mathrm{~nm}$ можно и другим способом, используя соотношения (1)-(3), как и для объемного расплава. Рассчитанные таким способом времена $T_{1 m}$ и $T_{1 Q}$ были близки к временам, приведенным в табл. 1 и 2.

Спин-решеточная релаксация изотопов галлия для сплава, введенного в пористое стекло с размером пор $7 \mathrm{~nm}$, как видно из табл. 1 и 2, а также из рис. 4, зависит от магнитного поля. Зависимость от магнитного поля показывает, что приближение быстрого движения в данном случае неприменимо. Поскольку на основании соотношения Корринги можно полагать, что вклад магнитной релаксации не изменился значительно по сравнению с объемным сплавом, то нарушение приближения быстрого движения обусловлено изменением квадрупольной релаксации, как и для ранее изученных наноструктурированных металлов и бинарных сплавов [4-6]. Согласно [4-6], общая формула для восстановления продольной ядерной намагниченности имеет вид:

$$
\begin{aligned}
\frac{M(t)}{M_{0}}= & 1-a\left[\frac{4}{5} \exp \left(-\frac{C \tau_{c} t}{1+4 \omega_{0}^{2} \tau_{c}^{2}}\right)\right. \\
& \left.+\frac{1}{5} \exp \left(-\frac{C \tau_{c} t}{1+\omega_{0}^{2} \tau_{c}^{2}}\right)\right] \exp \left(-\frac{t}{T_{1 m}}\right),
\end{aligned}
$$

где $M(t)$ и $M_{0}$ - зависящая от времени $t$ и равновесная намагниченности соответственно, $1-a-$ относительная намагниченность непосредственно после 180-градусного импульса, $\omega_{0}$ - ларморовская частота, $C$ и $\tau_{c}-$ квадрупольная постоянная и время корреляции атомного движения соответственно. Уравнения для релаксации изотопов ${ }^{71} \mathrm{Ga}$ и ${ }^{69} \mathrm{Ga}$ в разных полях содержат только два подгоночных параметра, $C^{71}$ и $\tau_{c}$, так как квадрупольные постоянные $C^{69}$ и $C^{71}$ для ${ }^{69} \mathrm{Ga}$ и ${ }^{71} \mathrm{Ga}$ связаны соотношением $C^{69}=C^{71} Q_{69}^{2} / Q_{71}^{2}$. Полученные подгоночные параметры $C^{71}$ и $\tau_{c}$ для сплава GaInSn в пористом стекле с порами $7 \mathrm{~nm}$ приведены в табл. 4 .

Для объемного расплава GaInSn и расплава в опаловой матрице и стекле с порами $18 \mathrm{~nm}$ также можно оценить параметры $C^{71}$ и $\tau_{c}$. В рамках приближения быстрого движения, учитывая неравенство $\omega_{0} \tau_{c} \ll 1$, уравнение (4) можно привести к виду

$$
\frac{M(t)}{M_{0}}=1-a \exp \left(-C \tau_{c} t\right) \exp \left(-\frac{t}{T_{1 m}}\right),
$$

откуда следует $T_{1 Q}^{-1}=C \tau_{c}$. Для объемного жидкого сплава время корреляции атомного движения можно принять равным времени корреляции в расплаве чистого галлия [4]. Эта величина приведена в табл. 4. Используя оценку для времени корреляции, по данным для времени квадрупольной релаксации можно оценить и квадрупольную постоянную $C^{71}$, также приведенную в табл. 4. Поскольку структура жидкого сплава не должна изменяться при введени в поры достаточно большого размера, можно предположить, что квадрупольная постоянная $C^{71}$ для расплава в порах опаловой матрицы и пористого стекла с размером пор $18 \mathrm{~nm}$ равна квадрупольной постоянной в объемном GaInSn. В рамках такого предположения по временам спиновой релаксации можно рассчитать время корреляции атомного движения для матриц с широкими порами. Результаты расчетов показаны в табл. 4.

В табл. 4 даны также значения отношения времени корреляции для жидкого GaInSn в пористых матрицах и в объемном расплаве. Как видно из табл. 4, время корреляции для GaInSn в опале и стекле с порами $18 \mathrm{~nm}$ выросло примерно в 5 раз. Влияние на время корреляции введения сплава в поры $7 \mathrm{~nm}$ оказалось значительно более сильным, что и обусловило нарушение приближения быстрого движения.

Разделение магнитного и квадрупольного вкладов в спиновую релаксацию ядер индия является более сложным. Хотя у индия есть два стабильных изотопа, ${ }^{115} \mathrm{In}$ и ${ }^{113} \mathrm{In}$, но их гиромагнитные отношения и квадрупольные моменты незначительно отличаются и использовать подход, рассмотренный выше для изотопов галлия, не 
представляется возможным [9]. В настоящей работе для разделения магнитного и квадрупольного вкладов в релаксацию изотопа ${ }^{115} \mathrm{In}$ и расчета квадрупольной постоянной $C^{115}$ мы воспользовались методом, предложенным в [6]. Для релаксации индия в объемном и наноструктурированном сплаве в рамках приближения быстрого движения можно написать систему уравнений

$$
\begin{gathered}
\left(T_{1}^{\text {bulk }}\right)^{-1}=C^{115} \tau_{c}^{\text {bulk }}+\left(T_{1 m}^{\text {bulk }}\right)^{-1}, \\
\left(T_{1}^{\text {opal }}\right)^{-1}=C^{115} \tau_{c}^{\text {opal }}+\left(T_{1 m}^{\text {bulk }}\right)^{-1}, \\
\left(T_{1}^{18 \mathrm{~nm}}\right)^{-1}=C^{115} \tau_{c}^{18 \mathrm{~nm}}+\left(T_{1 m}^{\text {bulk }}\right)^{-1},
\end{gathered}
$$

где $\tau_{c}^{\text {bulk }}, \tau_{c}^{\text {opal }}$ и $\tau_{c}^{18 \mathrm{~nm}}-$ времена корреляции атомного движения для объемного сплава, сплава в опале и сплава в пористом стекле с порами $18 \mathrm{~nm}$, рассчитанные выше по измерениям спиновой релаксации для ядер галлия. При составлении уравнений (5)-(6) было предположено, что структура расплава не изменяется при введении в опаловую матрицу и время магнитной релаксации для сплава в матрицах остается таким же, как и в объемном расплаве. В уравнениях (5)-(6) имеются только две неизвестные величины, $C^{115}$ и $T_{1 m}^{\text {bulk }}$. Численные оценки, полученные в рамках экспериментальной погрешности для времени продольной релаксации, дают для них значения $C^{115} \cong 190 \mu \mathrm{s}^{-2}$ и $T_{1 m}^{\text {bulk }} \cong 310 \mu \mathrm{s}$. В табл. 3 приведены также оценки для времени квадрупольной релаксации $T_{1 Q}=1 /\left(C^{115} \tau_{c}\right)$. Видно, что скорость квадрупольной релаксации ${ }^{115} \mathrm{In}$ значительно возрастает для наноструктурированного сплава за счет замедления атомного движения. Если в объемном сплаве основную роль играет магнитная релаксация, то для сплава в опале и стекле с порами $18 \mathrm{~nm}$ доминирует квадрупольная релаксация.

Как известно, время корреляции атомного движения в жидкости связано с коэффициентом диффузии $D$ соотношением $\tau=d^{2} / 6 D$, где $d-$ длина прыжка. Таким образом, проведенные исследования ядерной спиновой релаксации в тройном сплаве GaInSn показали значительное замедление диффузии в наноструктурированном расплаве, которое усиливалось с уменьшением размера пор.

\section{Список литературы}

[1] D.S. Evans, A. Prince. Metal. Sci. 12, 411 (1978).

[2] T.Y. Liu, P. Sen, C.J. Kim. J. Microelectromechan. Systems 21, 443 (2012).

[3] M. Knoblauch, J.M. Hibberd, J.C. Gray, A.J.E. van Bel. Nature Biotechnology 17, 906 (1999).

[4] E.V. Charnaya, T. Loeser, D. Michel, C. Tien, D. Yaskov, Y.A. Kumzerov. Phys. Rev. Lett. 88, 097602 (2002).

[5] D.Y. Podorozhkin, E.V. Charnaya, M.K. Lee, L.J. Chang, J. Haase, D. Michel, Y.A. Kumzerov, A.V. Fokin. Ann. Phys. 527, 248 (2015).

[6] E.V. Charnaya, C. Tien, M.K. Lee, Y.A. Kumzerov. J. Phys.: Condens. Matter 22, 195108 (2010).
[7] J. Kärger, D.M. Ruthven, D.N. Theodorou. Diffusion in Nanoporous Materials. Wiley-VCH (2012). 950 p.

[8] H. Konrad, J. Weissmuller, R. Birringer, C. Karmonik, H. Gleiter. Phys. Rev. B 58, 2142 (1998).

[9] J.M. Titman. Phys. Rep. 33, 1 (1977).

[10] A. Abragam. Principles of Nuclear Magnetism. Clarendon Press, Oxford (1989). 618 p.

[11] P.S. Hubbard. J. Chem. Phys. 53, 985 (1970).

[12] T. Tokuhiro. J. Magn. Res. 76, 22 (1988).

[13] Д.Ю. Подорожкин, Е.В. Чарная, D. Michel, J. Haase, C. Tien, M.K. Lee, L.J. Chang, Ю.А. Кумзеров. ФТT 54, 1035 (2012). 